\title{
Paranasal sinus disease in HIV antibody positive patients
}

\author{
Alison Grant, Marcelle von Schoenberg, H R Grant, R F Miller
}

\begin{abstract}
Objective-To investigate the prevalence of radiologically-diagnosed paranasal sinus disease in HIV-1 seropositive patients.

Subjects and setting-476 patients admitted to a dedicated inpatient unit for HIV and AIDS at the Middlesex Hospital, London, between September 1988 and February 1992.

Design-Retrospective review of patients' case notes and radiological records.

Results-30 patients $(6 \cdot 3 \%)$ had radiological evidence of paranasal sinus disease. At the time of admission, sinusitis was in the differential diagnosis in only 12 of the 30 patients; 13 patients were initially diagnosed as having meningitis. Pseudomonas aeruginosa was the causative organism in four patients, all of whom had advanced HIV disease. All four responded to appropriate antibiotics but had early relapse of infection.

Conclusions-Sinusitis is an important and under-recognised cause of morbidity in patients with HIV disease.
\end{abstract}

(Genitourin Med 1993;69:208-212)

\section{Introduction}

Although an increased susceptibility to bacterial infections has been well described in patients with HIV disease there have been few reports of sinusitis. ${ }^{1}$ Recently we have seen several HIV seropositive patients presenting with severe sinus disease, in four of whom Pseudomonas aeruginosa, a rare cause of sinusitis in the immunocompetent, was the causative organism

In this paper we describe our experiences of managing sinusitis in a cohort of HIV seropositive patients admitted to the Middlesex Hospital over a 42 month period and suggest a policy for the management of this condition.

\section{Methods}

Case finding

All HIV positive patients at the Middlesex Hospital are admitted to a dedicated unit under a single consultant. We reviewed the radiological records of all these patients over a 42 month period (September 1988 to February 1992) and identified all patients who had radiological abnormalities of the sinuses on the basis of sinus radiographs, CT or magnetic resonance imaging of the brain.

\section{Data analysis}

We examined the corresponding case notes and recorded the following information: age, sex and risk factors for HIV infection; risk factors for sinus disease; presenting symptoms; laboratory investigations and outcome.

\section{Clinical classification}

We defined sinusitis as one or more of the following symptoms: facial pain, rhinorrhoea, post-nasal drip or nasal obstruction due to underlying inflammation of the nasal or paranasal sinus mucosa. We classified the clinical severity of sinusitis as follows: (i) Asymptomatic: radiological evidence of sinus disease but either asymptomatic or symptoms attributable to another identified pathology, (ii) Mild: symptomatic sinus disease which was self-limting and did not require any specific intervention with antibiotics or antihistamines, (iii) Moderate: symptomatic disease which was not self-limiting and required treatment, but responded to conventional measures, (iv) Severe: symptomatic disease that either persisted after a standard course of treatment or required operative intervention.

\section{Radiological classification}

We devised a system for classifying the radiological severity of sinus disease by assessing four radiological parameters in ascending order of clinical significance: a higher score indicates more severe disease (table 1).

\section{Results}

Between September 1988 and February 1992, 476 HIV seropositive patients were admitted to the Middlesex Hospital: of these, $30 \mathrm{had}$ radiological evidence of sinus disease.

Table 1 Radiological classification of sinus disease

\begin{tabular}{lc}
\hline Radiological abnormality & Score \\
\hline Mucosal thickening & 1 point \\
Fluid level & 4 points \\
Opaque sinus & 8 points \\
Complications: & 16 points \\
Orbital abscess & 16 points \\
Osteomyelitis & 32 points \\
Intracranial abscess &
\end{tabular}

Radiological severity was calculated from the sum of the score for each abnormality for each sinus (right and left maxillary, ethmoid, sphenoid and frontal sinuses respectively). A total score of $1-7=$ mild, $8-15=$ moderate and $>16=$ severe radiological disease.
Middlesex Hospital, WiN 8AA UK

Accepted for publication 18 December 1992 
Sinus radiographs were performed on another nine patients who presented with headache, and were normal. Two further patients had skull radiographs performed following a head injury, and these showed no evidence of sinus disease. These 11 patients were excluded from the subsequent analysis.

\section{Patient characteristics}

The median age was 34 years (range 22 to 56). Twenty-seven were male, of whom 21 were homosexual, four were intravenous drug users, one was African and three had no apparent risk factor. Two men were both homosexual and intravenous drug users. Of the three women, one was an intravenous drug user and two were African. Twenty-three patients had a previous AIDS-defining diagnosis.

\section{Risk factors for sinus disease}

Six patients had a past history of sinusitis. Four patients had a history of asthma, one with coincident bronchiectasis, and one (an intravenous drug user) had bronchiectasis without a history of atopy. Ten patients were cigarette smokers and 20 were non-smokers or ex-smokers.

\section{Drug therapy}

Five patients were taking long term oral steroids (for immune thrombocytopaenia, oedema due to cutaneous Kaposi's sarcoma, chronic asthma, myositis and to improve well-being respectively). Twelve patients were taking continuous antibiotics orally: co-trimoxazole (9) and dapsone (1) as prophylaxis against Pneumocystis carinii pneumonia; rifampicin, isoniazid and pyrazinamide for treatment of tuberculosis (2); erythromycin (1) and doxycycline (1) for recurrent bronchitis. Nine patients were receiving monthly nebulised pentamidine. Four patients were taking zidovudine and two ganciclovir, but all six had neutrophil counts greater than $0.6 \times$ $10^{9}$ per litre.

\section{Laboratory investigations}

One patient had severe neutropaenia; his only medication was co-trimoxazole $960 \mathrm{mg}$ daily. The median white cell count was 3.3 (range $0.6-15 \cdot 7) \times 10^{9}$ per litre and the median lymphocyte count was 0.6 (range $0 \cdot 28-2 \cdot 1$ ) $\times 10^{9}$ per litre. A CD4 count was available in 19 patients: the median was 0.02 (range $0-0.78) \times 10^{9}$ per litre (normal range = $0 \cdot 35-2 \cdot 2 \times 10^{9}$ per litre).

\section{Clinical presentation}

Of the 30 patients, 16 had specific symptoms of sinus disease (usually unilateral facial pain). 11 others had non-specific symptoms, usually symmetrical headache, and the remaining three patients were asymptomatic.

In 13 patients a clinical diagnosis of meningitis was made initially, and of these 10 had a lumber puncture performed to exclude this diagnosis. Of the three patients in whom lumbar puncture was not carried out, the reasons were the presence of a mass lesion on cranial imaging, spontaneous resolution of the headache and refusal to undergo the procedure respectively. Two patients were diagnosed as having meningitis on the basis of cerebrospinal fluid examination, but in the remaining eight sinusitis was the most likely cause of the presenting symptoms.

Twelve of the 30 patients had a clinical diagnosis of sinusitis made at the time of admission.

We present two case histories to illustrate some of the problems caused by severe sinus disease.

Case 1

A 56 year old Caucasian homosexual man presented with a 5 day history of frontal headache, fever and nasal congestion. Ten months previously he had had Pneumocystis carinii pneumonia: at this time his $\mathrm{CD} 4$ count was 0.06 (normal range $0.35-2.2$ ) $\times 10^{9}$ per litre. Subsequently zidovudine $250 \mathrm{mg}$ qds and nebulised pentamidine $300 \mathrm{mg}$ monthly were commenced, and he had remained well in the intervening period. On examination he was febrile at $38^{\circ} \mathrm{C}$ and neurological examination was normal. Cryptococcal meningitis was suspected. Prior to lumbar puncture, CT of the brain was performed, which showed diffuse cortical atrophy. Microscopy of the cerebrospinal fluid obtained at lumbar puncture was normal and culture was negative. At this point sinusitis was suspected, and so sinus radiographs were peformed, revealing bilateral maxillary fluid levels. He was treated with oral amoxycillin and ephedrine nose drops but his symptoms persisted: a second lumbar puncture was performed and cerebrospinal fluid examination was again normal. In view of his persisting symptoms of sinusitis, a right antral wash-out was performed. Culture of lavage fluid revealed a mixed growth of organisms including Pseudomonas aeruginosa, sensitive to polymixin, amikacin, ciprofloxacin, azlocillin, ceftazidime and gentamicin. Follow-up sinus radiographs showed a pansinusitis (fig 1). Chloramphenicol was commenced. The patient improved but his fever returned when chloramphenicol was stopped. He was therefore given oral ciprofloxacin, but after completing this second course of antibiotics his symptoms of headache and nasal congestion recurred, and so he was maintained on continuous antibiotics after discharge.

Six months later the patient was readmitted with a 10 day history of severe frontal headache and retro-orbital pain. A CT scan of the sinuses showed right ethmoidal sinusitis with erosion of the medial orbital wall (fig 2). Sputum culture revealed Pseudomonas aeruginosa with the same sensitivity pattern as found previously. After seven days of intravenous azlocillin and gentamicin there was clinical improvement, but when antibiotics were stopped he deteriorated rapidly. To promote resolution of infection his ethmoid air cells were drained under direct transnasal endoscopic vision with good results. In the postoperative period he went into renal failure and he died 3 weeks later. 
Figure 1 Case 1: Sinus radiographs at original presentation showing bilateral maxillary fluid levels and opaque right frontal sinus.

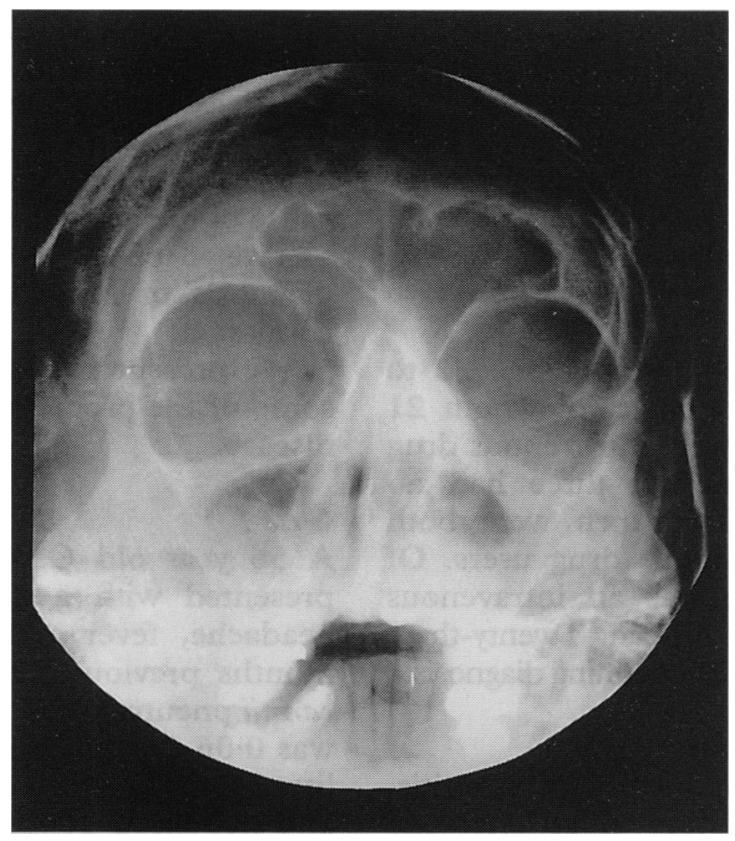

Case 2

A 35 year old Caucasian homosexual man presented with a two day history of left periorbital pain, purulent nasal discharge, postnasal drip, fever and pain on swallowing. $\mathrm{He}$ also described transient sensory symptoms in his left arm. In the six weeks prior to this admission he had been hospitalised several times because of recurrent bacterial chest infections due to Pseudomonas aeruginosa. Treatment had been complicated by the development of hypersensitivity reactions to several antibiotics.

On examination he was unwell and febrile at $37 \cdot 5^{\circ} \mathrm{C}$. There were no abnormal neurological signs. In view of his sensory symptoms, magnetic resonance imaging of the head was performed: this showed no intracerebral abnormality but showed mucosal thickening and a fluid level in the left maxillary antrum, mucosal thickening of both ethmoid sinuses and the right sphenoid sinus and abnormal signal from the left maxilla suggestive of osteomyelitis (figs 3 and 4). A chest radiograph showed a small cavitating

Figure 2 Case 1: CT scan on re-presentation showing right ethmoidal sinusitis with erosion of the medial orbital wall (arrows). lesion in the right upper zone. The CD4 count was severely reduced at $0.01 \times 10^{9}$ per litre. A left antral wash-out revealed mucopurulent fluid which on culture yielded a heavy growth of Pseudomonas aeruginosa, as did samples of spontaneously expectorated sputum. These isolates were sensitive to polymixin, amikacin, ciprofloxacin, azlocillin, ceftazidime and gentamicin. Bronchoalveofär lavage was performed in order to exclude p $\mathrm{pl}$ monary tuberculosis, and lavage fluid afso yielded Pseudomonas aeruginosa on cultume, with the same pattern of antibiotic sensitivity as the previous isolates. Oesophageal candiäiasis was found on endoscopy. The patient improved on intravenous azlocillin and gentamicin, but he developed a diffuse drug rash after nine days and so his antibiotics were stopped. Four days later his symptoths returned, and he was treated with intravengits aztreonam and tobramycin to which he responded rapidly. After ten days of tregtment he again developed a drug rash amd antibiotics were stopped. There was no recurrence of his symptoms and he was subsequently discharged home.

\section{Clinical severity}

Six patients were asymptomatic, eight häd mild disease, 10 had moderate disease and six had severe disease. Of those with severe disease, four patients required operative intigvention. One patient had bilateral intranasal antrostomies to facilitate sinus drainage ag d resolution of infection. Three patients unds went antral wash-outs, one requiring the p procedure twice because of persisting symptorŏ this latter patient ultimately underwent fuß tional endoscopic sinus surgery.

\section{Imaging}

Sixteen patients had plain sinus radiographs, 11 patients had CT of the head or the sinuses and 16 patients had magnetic resonarice

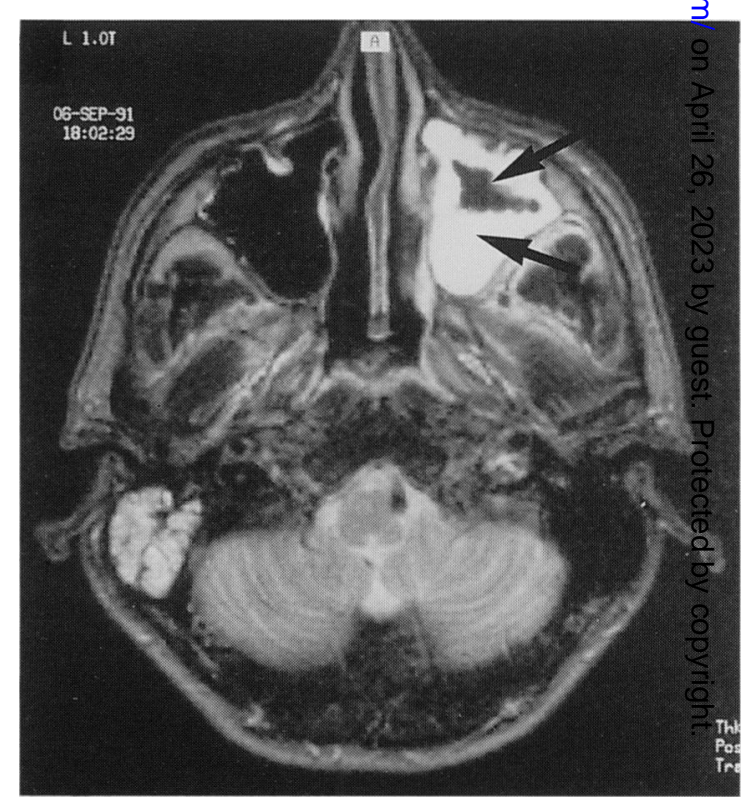

Figure 3 Case 2: T2-weighted magnetic resonance image showing mucosal thickening and a fluid level maxillary antrum, and abnormal signal from the left maxilla (arrows). 
Figure 4 Case 2: T2-weighted magnetic resonance image showing bilateral ethmoidal and right sphenoidal mucosal thickening.

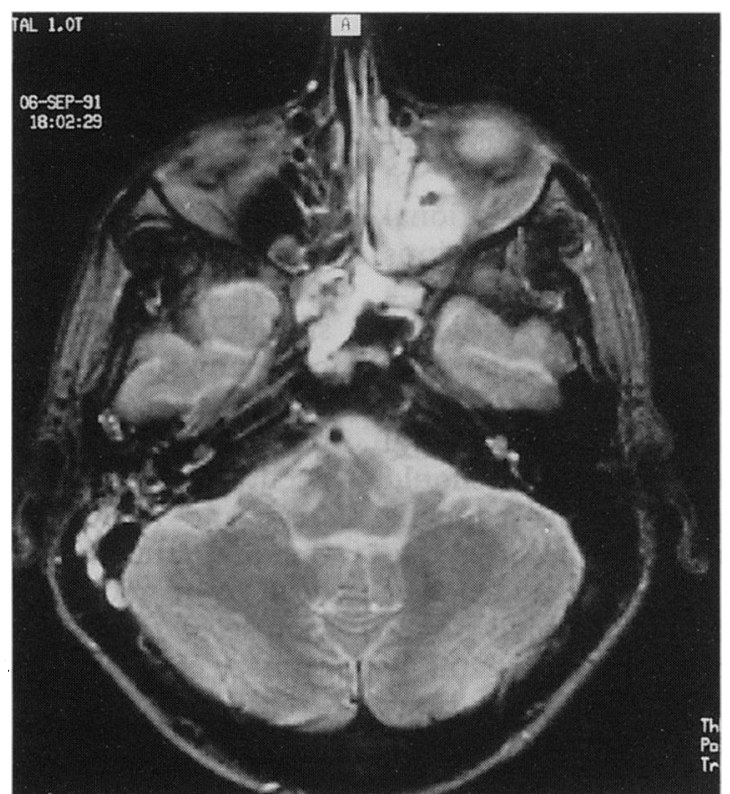

imaging studies. Using the scoring system described, 19 patients had mild disease, six had moderate disease and five had severe radiological disease. Table 2 shows the correlation between the clinical and the radiologically-defined severity of sinusitis.

\section{Bacteriology}

Six patients had positive microbiological cultures relevant to their sinus disease. Pseudomonas aeruginosa was isolated in four patients, all of whom had advanced HIV disease. In all four cases the infection was community-acquired. These isolates were from sputum (1 patient); sputum, post-nasal space biopsy and blood culture ( 1 patient); sputum, bronchoalveolar lavage fluid and sinus washings ( 1 patient) and sinus washings ( 1 patient). The sensitivity pattern of the

Table 2 Correlation of clinical and radiological severity of sinusitis

\begin{tabular}{llll}
\hline & \multicolumn{2}{l}{ Radiological severity } \\
\cline { 2 - 4 } Clinical severity & Mild & Moderate & Severe \\
\hline Asymptomatic & 4 & 2 & 0 \\
Mild & 6 & 2 & 0 \\
Moderate & 9 & 0 & 1 \\
Severe & 0 & 2 & 4 \\
\hline
\end{tabular}

Table 3 Outcome of ten patients specifically treated for sinusitis

\begin{tabular}{|c|c|c|c|}
\hline Patient & Organism & Initial therapy & Outcome \\
\hline $\begin{array}{l}1 \\
2\end{array}$ & & $\begin{array}{l}\mathrm{AB} \\
\mathrm{AB} \text { and } \\
\text { bilateral AWO }\end{array}$ & $\begin{array}{l}\text { Improved, no recurrence } \\
\text { Improved, no recurrence }\end{array}$ \\
\hline 3 & Ps. aeruginosa & $\mathbf{A B}$ & $\begin{array}{l}\text { Persistent symptoms, required } \\
\text { AWO twice. Further recurrence } \\
\text { at } 6 \text { months. }\end{array}$ \\
\hline $\begin{array}{l}4 \\
5 \\
6\end{array}$ & $\begin{array}{l}\text { H. influenzae } \\
\text { S. aureus } \\
\text { Ps. aeruginosa }\end{array}$ & $\begin{array}{l}\mathrm{AB} \\
\mathrm{AB} \text { and BDNA } \\
\mathrm{AB} \text { and left } \mathrm{AWO}\end{array}$ & $\begin{array}{l}\text { Improved, no recurrence } \\
\text { Improved, no recurrence } \\
\text { Relapsed after } 1 \text { course of } \\
\text { antibiotics, better after } \\
\text { second course }\end{array}$ \\
\hline $\begin{array}{l}7 \\
8\end{array}$ & Ps. aeruginosa & $\begin{array}{l}\mathbf{A B} \\
\mathbf{A B}\end{array}$ & $\begin{array}{l}\text { Partial improvement of symptoms } \\
\text { Partial improvement of symptoms, } \\
\text { recurrence at } 2 \text { months }\end{array}$ \\
\hline $\begin{array}{r}9 \\
10\end{array}$ & & $\begin{array}{l}\mathbf{A B} \\
\mathbf{A B}\end{array}$ & $\begin{array}{l}\text { Improved, no recurrence } \\
\text { Improved, recurrence at } 3 \text { months }\end{array}$ \\
\hline
\end{tabular}

AB - antibiotics, AWO-antral washout, BINA-bilateral intranasal antrostomies. organisms was the same in all four patients, as described in the case histories.

Two further patients had positive microbiological cultures: one had Haemophilus influenzae isolated from the sputum and one had Staphylococcus aureus isolated from sinus washings.

\section{Intervention}

Ten patients received specific treatment for sinusitis: all 10 were given $0.5 \%$ ephedrine nose drops for symptom relief, and two also received systemic antihistamines. All 10 were commenced on antimicrobial therapy, five by the intravenous route and five orally. Four patients required operative intervention. Thirteen patients received broad spectrum antibiotics primarily with the intention of treating infection at another site which is likely to have had an additional beneficial effect on the observed sinus disease. In seven patients there was no specific intervention.

\section{Outcome}

The outcome in those patients treated specifically for sinusitis is shown in table 3 . There was recurrent disease in one of the six asymptomatic patients, two of eight with mild disease, 3 of 10 with moderate disease and four of six who had severe disease.

\section{Discussion}

Only four patients underwent surgical procedures, and those procedures that were performed were relatively minor. This compares with intervention rates reported by other groups of four out of $75^{2}$ and 18 out of $68 .^{3}$ As a definite bacteriological diagnosis was made in only a few patients, we suggest that early surgical intervention might both provide the diagnosis and alleviate symptoms. The benefit of early intervention is illustrated by the case histories.

Previous authors have differed in their strategy for the management of sinusitis in patients with AIDS, particularly with respect to operative intervention. ${ }^{6} \mathrm{We}$ concur with the view that if sinusitis does not respond to initial treatment with standard antibiotics such as doxycycline then a sinus wash-out should be performed in order to make a bacteriological diagnosis and enable rational antibiotic therapy. ${ }^{4}$ This is particularly important because in addition to Pseudomonas aeruginosa, a wide variety of unusual organisms have been described as a cause of sinusitis in patients with AIDS, including Cryptococcus neoformans and cytomegalovirus, ${ }^{7}$ infections which would not respond to standard broad-spectrum antibiotics.

In an HIV seropositive patient who presents with headache meningitis must be excluded, and cranial imaging (with CT or magnetic resonance imaging) and a lumbar puncture are needed to rule out this diagnosis. Once meningitis has been excluded, sinusitis should be considered in the differential diagnosis. Plain sinus radiographs should be performed to confirm the diagnosis: if 
these are normal, sinusitis is unlikely. In a study of 54 immunocompetant patients presenting with symptoms and signs of sinusitis, only $9 \%$ of patients with normal sinus radiographs had bacteriological evidence of sinusitis on antral wash-out. ${ }^{8}$ Conventional axial CT may not detect the presence of sinus disease; coronal scans may be required. If a patient with sinusitis is not systemically unwell and there is no clinical evidence of complications such as orbital cellulitis or intracranial infection, a 10-day course of oral antibiotics (for example doxycycline) should be given. Failure to respond to this regime is an indication for coronal CT of the sinuses to assess disease extent and look for evidence of osteomyelitis of the ethmoids, orbital bones or maxillae, or intracranial sepsis, particularly a frontal lobe abscess; at this stage sinus wash-outs should be performed to make a bacteriological diagnosis.

If the patient is systemically unwell, admission is recommended for a sinus wash-out and intravenous antibiotics. If there is no improvement after 48 hours of treatment, or if there are complications of sinus disease (orbital cellulitis or intracranial infection), coronal CT of the sinuses should be performed to identify the extent and severity of the disease and assess the need for further surgical intervention.

Sinusitis due to Pseudomonas aeruginosa may require a prolonged course of antibiotics, because of the tendency to relapse after shorter courses of antibiotics and the potential for osteomyelitis. This is analagous to the treatment of malignant otitis externa, a chronic osteomyelitis of the temporal bone in immunocompromised patients due to infection of the external ear canal by Pseudomonas aeruginosa. ${ }^{9}$

Sinusitis is rarely included in the differential diagnosis of headache in HIV seropositive patients. Surgical intervention may be required either to make a microbiological diagnosis or as a therapeutic procedure to relieve symptoms. An urgent ENT opinion should be sought in patients who are systemically unwell in order that sinus wash-outs or other surgical intervention may be carried out. Sinusitis due to Pseudomonas aeruginosa occurs in the context of advanced HIV disease, and responds to specific antimicrobial therapy, but the course of treatment may need to be prolonged in order to prevent the relapse of symptoms and signs or the development of chronic infection.

1 Rubin JS, Honigberg R. Sinusitis in patients with the acquired immunodeficiency syndrome. Ear Nose Throa 于 1990;69:460-3

2 Zurlo J, Feuerstein IM, Lebovics R, Lane HC. Sinusitis in HIV-1 infection. Am f.Med 1992;93:157-62.

3 Godofsky EW, Zinreich J, Armstrong M, Leslie JM, Weikel CS. Sinusitis in HIV-infected patients: a clinical and radiographic review. Am $\mathcal{F}$ Med 1992;93:163-70.

4 Poole MD, Postma D, Cohen MS. Pyogenic otorhinologic infections in acquired immune deficiency syndrome. infections in acquired immune

5 Schrager LK Bacterial infections in AIDS patients. AIDS 1988;2 (Suppl 1):S183-9.

6 Lamprecht J, Wiedbrauk C. Sinusitis und andere typische Erkrankungun im HNO-Bereich im Rahmen des erworbenen Immundefekt-Syndroms (AIDS). HNO 1988;36: 489-92.

7 Meiteles LZ, Lucente FE. Sinus and nasal manifestations of the acquired immunodeficiency syndrome. Ear Nose Throat $₹$ 1990;69:454-9.

8 Pfleiderer AG, Drake Lee AB, Lowe D. Ultrasound of the sinuses; a worthwhile procedure? A comparison of ultrasound and radiography in predicting the findings. Cin Otolaryngol 1984;9:335-41.

Hammond V. Diseases of the external ear. In: Kerr AG ed. Scott-Brown's Otolaryngology. London: Butterworths, 1987;3:168-9. 